\title{
Reduced Intensity Bone Marrow Transplantation with Post-Transplant Cyclophosphamide for Pediatric Inherited Immune Deficiencies and Bone Marrow Failure Syndromes
}

\author{
Orly R. Klein ${ }^{1}$ (D) - Samantha Bapty ${ }^{2} \cdot$ Howard M. Lederman $^{3} \cdot$ M. Elizabeth M. Younger ${ }^{3} \cdot$ Elias T. Zambidis ${ }^{1}$. \\ Richard J. Jones ${ }^{1} \cdot$ Kenneth R. Cooke ${ }^{1} \cdot$ Heather J. Symons ${ }^{1}$
}

Received: 5 June 2020 / Accepted: 22 October 2020 / Published online: 6 November 2020

(C) Springer Science+Business Media, LLC, part of Springer Nature 2020

\begin{abstract}
Purpose Allogeneic bone marrow transplantation (alloBMT) is the only cure for many primary immune deficiency disorders (PIDD), primary immune regulatory disorders (PIRD), and inherited bone marrow failure syndromes (IBMFS).

Methods We report the results of 25 patients who underwent alloBMT using reduced intensity conditioning (RIC), alternative donors, and post-transplantation cyclophosphamide (PTCy). In an attempt to reduce regimen-related toxicities, we removed lowdose TBI from the prep and added mycophenolate mofetil and tacrolimus for graft-versus-host disease (GVHD) prophylaxis for all donor types in the latter 14 patients. Donors were haploidentical related $(n=14)$, matched unrelated $(n=9)$, or mismatched unrelated $(n=2)$. The median age was 9 years (range 5 months -21 years).

Results With a median follow-up of 26 months (range 7 months-9 years), the 2-year overall survival is $92 \%$. There were two deaths, one from infection, and one from complications after a second myeloablative BMT. Three patients developed secondary graft failure, one at 2 years and two at $>3$ years, successfully treated with CD34 cell boost in one or second BMT in two. The remaining 20 patients have full or stable mixed donor chimerism and are disease-free. The incidence of mixed chimerism is increased since removing TBI from the prep. The 6-month cumulative incidence of grade II acute GVHD is 17\%, with no grade III-IV. The 1-year cumulative incidence of chronic GVHD is $14 \%$, with severe of 5\%.

Conclusion This alloBMT platform using alternative donors, RIC, and PTCy is associated with excellent rates of engraftment and low rates of GVHD and non-relapse mortality, and offers a curative option for patients with PIDD, PIRD, and IBMFS.

Trial registration ClinicalTrials.gov Identifier: NCT04232085
\end{abstract}

Keywords Blood and marrow transplantation · primary immune deficiency disorders · primary immune regulatory disorders . inherited bone marrow failure disorders $\cdot$ alternative donor $\cdot$ post-transplant cyclophosphamide

Heather J. Symons

hsymons2@jhmi.edu

1 Hematologic Malignancies and Blood and Marrow Transplantation Program, Sidney Kimmel Comprehensive Cancer Center, The Johns Hopkins University School of Medicine, Baltimore, MD, USA

2 Department of Pediatrics, Children's Hospital Los Angeles, Los Angeles, CA, USA

3 Division of Allergy and Immunology, Department of Pediatrics, The Johns Hopkins University School of Medicine, Baltimore, MD, USA

\section{Introduction}

Allogeneic blood and marrow transplantation (alloBMT) is curative for many primary immune deficiency disorders (PIDD), primary immune regulatory disorders (PIRD), and inherited bone marrow failure syndromes (IBMFS). The best outcomes have used HLA-matched sibling donors and myeloablative conditioning (MAC); however, only $30 \%$ of children have an HLA-matched sibling [1]. The use of donors other than an HLA-matched sibling has historically been limited by high rates of transplantrelated mortality (TRM) secondary to graft failure, infection, and acute and chronic graft-versus-host disease (GVHD) [2-7]. Reduced intensity conditioning (RIC) regimens are preferred in children with nonmalignant 
disorders as myeloablation offers no direct disease benefit, and RIC offers less short- and long-term morbidity. However, graft failure with RIC remains a critical obstacle, particularly with donors other than HLA-matched. A platform that expands the donor pool, optimizes engraftment, and minimizes toxicities for PIDD/PIRD/IBMFS patients would represent a significant advancement.

We have previously reported our promising singleinstitution pilot experience using RIC and alternative donors with post-transplant cyclophosphamide (PTCy) for a small cohort of PIDD/PIRD/IBMFS patients $(n=11)$ [8]. Successful engraftment and low rates of GVHD led us to offer this platform to additional patients with two major changes. First, in an attempt to reduce regimen-related toxicity, we removed the previously included low-dose total body irradiation (TBI) from the conditioning regimen. Second, in addition to PTCy, post-transplant mycophenolate mofetil (MMF) and tacrolimus were included for all donor types to minimize GVHD even further.

A uniform, "one size fits all" regimen for rare, heterogeneous diagnoses has precedence in the literature $[4,9,10]$, and offers a safe and effective curative option for any PIDD/PIRD/ IBMFS patient in need of a BMT, using a platform with a proven track record. We herein report our expanded institutional experience treating pediatric patients with lifethreatening PIDD/PIRD/IBMFS with longer-term follow-up, including additional patients treated without TBI and with MMF and tacrolimus, where we observed a higher incidence of mixed chimerism in the PIDD/PIRD but not the IBMFS patients, and a lower cumulative incidence of GVHD in patient with 10/10 HLA-matched unrelated donors.

\section{Materials and Methods}

\section{Patients}

This retrospective study was approved by the institutional review board of The Johns Hopkins Hospital (JHH). All pediatric patients (age 1 month to 21 years) who underwent alloBMT at JHH from January 2009 to January 2019 for specified nonmalignant conditions (PIDD, PIRD, and IBMFS), and who did not have a matched sibling donor, were included. BMTs were performed as standard of care, and all patients/ guardians provided informed consent for treatment.

Alternative donors included matched unrelated donors (MUD), mismatched unrelated donors (MMUD), or haploidentical related (haplo) donors. Inclusion criteria included adequate organ function testing and Karnofsky or Lansky performance status $\geq 60 \%$. Exclusion criteria included positive leukocytotoxic crossmatch to mismatched donor HLA and uncontrolled infection. Data from the medical record including patient demographics, transplant and clinical data, complications, laboratory and radiologic studies, therapy received, overall outcomes, and transplant-related complications were abstracted and reviewed.

\section{Regimen}

In our previously reported patients $(n=11)$ [8], the preparative regimens varied. All of the newly reported patients received the same uniform regimen with alemtuzumab, fludarabine, and melphalan (Fig. 1), notably, without TBI and with posttransplant MMF and tacrolimus for all donor types. One newly reported patient did have TBI included on day -1 .

Alemtuzumab dosing was based on weight, with children $>10 \mathrm{~kg}$ receiving a total of $48 \mathrm{mg}$ intravenously (IV) over 3 days, starting on day -14 , with an initial dose of $3 \mathrm{mg}$, followed by doses of $10 \mathrm{mg} / 15 \mathrm{mg} / 20 \mathrm{mg}$. Patients $<10 \mathrm{~kg}$ received a total of $33 \mathrm{mg}$, with a test dose of $3 \mathrm{mg}$ followed by $10 \mathrm{mg} / 10 \mathrm{mg} / 10 \mathrm{mg}$. All patients received fludarabine $150 \mathrm{mg} / \mathrm{m}^{2}$ divided over 5 days (or $1 \mathrm{mg} / \mathrm{kg}$ /day for 5 days for patients $<10 \mathrm{~kg}$ ), starting on day -8 . The dosing for melphalan was $140 \mathrm{mg} / \mathrm{m}^{2}$ (or $3.4 \mathrm{mg} / \mathrm{kg}$ for patients $<10 \mathrm{~kg}$ ), either given as a single dose on day -2 or equally divided over 2 days on days -3 and -2 . In some patients, TBI of 200 cGy was given on day -1 .

GVHD prophylaxis consisted of PTCy, mycophenolate mofetil (MMF), and tacrolimus for all newly reported patients, regardless of donor type. In our previously reported patients [8], patients who received 10/10 HLA-matched unrelated donors (MUDs) $(n=4)$ received PTCy alone for GVHD prophylaxis, and patients who received haploidentical donors or mismatched unrelated donors received PTCy, MMF, and tacrolimus. Cyclophosphamide was given IV on days +3 and +4 , at a dose of $50 \mathrm{mg} / \mathrm{kg} /$ day (excepting the two DKC patients, who received only one dose on day +3 ), followed by MMF $15 \mathrm{mg} / \mathrm{kg} / \mathrm{dose}$ IV or by mouth TID (maximum daily dose $3 \mathrm{~g} /$ day), and tacrolimus $0.015 \mathrm{mg} / \mathrm{kg} /$ dose IV every $12 \mathrm{~h}$, both starting on day +5 . MMF was stopped on day +35 . Tacrolimus was switched to oral as tolerated, and dose adjusted to maintain a trough between 5 and $10 \mathrm{ng} / \mathrm{mL}$. Tacrolimus was tapered starting on day +150 and discontinued by day + 180 if there was stable donor chimerism and no active GVHD.

\section{Donor Selection}

Donors were molecularly typed at HLA-A, -B, -Cw, DRB1, and -DQB1. Unrelated donors were matched at either $5 / 8$ (DQB1 matching was not available) or 9 or 10/10. Haploidentical donors were 1st degree relatives or half-sibling who had one to five mismatches at the antigen or allele level. Donors were chosen based on age, gender, CMV status, medical comorbidities (for family members), and availability. Preference was given equally to $10 / 10$ 


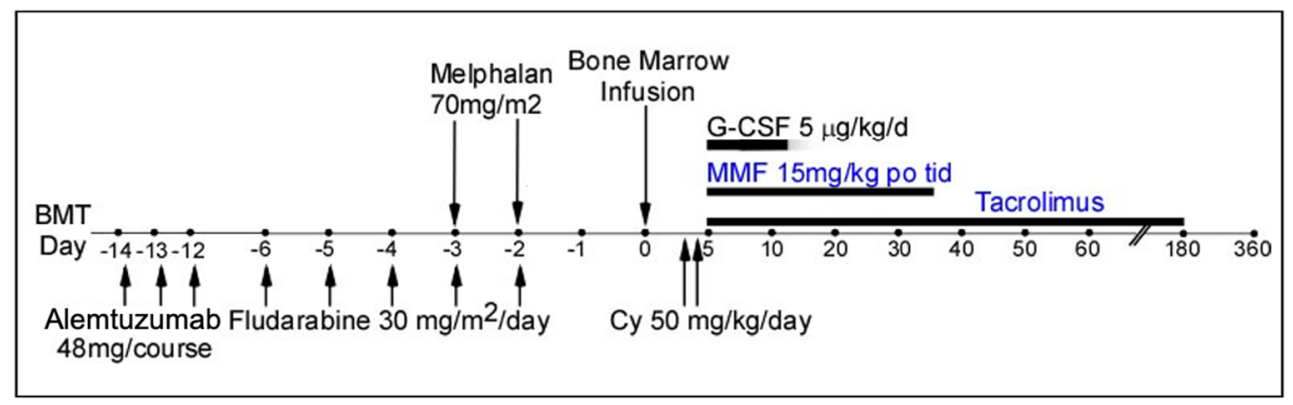

Fig. 1 Preparatory regimen and GVHD prophylaxis. Prep includes alemtuzumab, fludarabine, and melphalan. GVHD prophylaxis includes cyclophosphamide, tacrolimus, and mycophenolate mofetil. Cy, cyclophosphamide; GCSF, granulocyte colony stimulating factor; MMF, mycophenolate mofetil

characterized as early (before day +365 ) or late (at or after day +365$)$.

Acute GVHD (aGVHD) was graded per standard criteria [11], and chronic GVHD (cGVHD) was graded per NIH Working Group Reports $[12,13]$. Invasive fungal infections were defined according to published guidelines [14]. Overall survival (OS) was defined as alive, and event-free survival (EFS) was defined as alive without graft failure. Disease-free survival (DFS) was defined as alive, with any detectable donor chimerism and without any symptoms of the underlying disease. Transplant-related mortality (TRM) was defined as death related to the transplant.

\section{Statistical Analysis}

Descriptive statistics were used to summarize baseline patient and transplant characteristics. The probability of OS was estimated using the Kaplan-Meier method with 95\% confidence intervals (CIs) [15]. Cumulative incidences of relapse, TRM, and GVHD were estimated by competing-risk analysis using Gray's method [16]. Relapse and TRM were competing risks for each other. Death, relapse, and graft failure were competing risks for GVHD. Data were analyzed with the Prism version 8.2.0 (Graphpad Software, La Jolla, CA).

Neutrophil recovery was defined as the number of days from BMT to the first of 3 consecutive days with an absolute neutrophil count above $0.5 \times 10^{9} / \mathrm{L}$. Platelet recovery was defined as platelet count greater than $20 \times 10^{9} / \mathrm{L}$ for 3 consecutive days without transfusion in the preceding 7 days. Routine donor chimerism analysis was performed on days $+30,+$ $60,+90,+180$, and 1 and 2 years after BMT. Chimerism was analyzed in whole blood (WB) and isolated CD3+ cells (TCs). Mixed chimerism was defined as $\geq 5 \%$ and $<95 \%$ donor cells, and full chimerism as $\geq 95 \%$ donor cells, in WB. Primary graft failure was defined as $<5 \%$ donor chimerism in WB by day +60 . Secondary graft failure was defined as loss of donor engraftment $(<5 \%$ donor chimerism in WB) after initially achieving neutrophil recovery and detection of donor chimerism. Secondary graft failure was further

\section{Results}

\section{Patient, Donor, and Graft Characteristics}

Twenty-five patients were treated; donor and graft characteristics are summarized in Tables 1 and 2. The median followup of surviving patients is 26 months (ranging 7 months9 years). None of the patients had detectable donor-specific anti-HLA antibodies at the time of BMT. Bone marrow was the preferred stem cell source for all patients; 24 patients (96\%) received a bone marrow graft, and one patient received a PBSC graft because of donor preference. 
Table 1 Patient, donor, and allograft characteristics

\begin{tabular}{lll}
\hline Characteristic & Number & Percent \\
\hline
\end{tabular}

Age at BMT, years

$\begin{array}{ll}\text { Median } & 9 \\ \text { Range } & 0.4-21 \\ \text { Diagnosis } & \\ \text { CGD } & 11 \\ \text { Birc4/XIAP deficiency } & 2 \\ \text { DBA } & 2 \\ \text { DKC } & 2 \\ \text { Hyper-IgM } & 1 \\ \text { IPEX } & 1 \\ \text { HLH } & 1 \\ \text { GT } & 1 \\ \text { CVID } & 1 \\ \text { CTLA4 deficiency } & 1 \\ \text { STAT3 gain-of-function } & 1 \\ \text { ADA-deficient SCID } & 1 \\ \text { Male recipients } & 19 \\ \text { Graft source } & \\ \text { Bone marrow } & \\ \text { Mobilized peripheral blood stem cells } & 1 \\ \text { Donor source } & \end{array}$

Donor source

HLA haploidentical related

10/10 HLA-matched unrelated

9/10 HLA-matched unrelated

5/8 HLA-matched unrelated

Relationship of haploidentical donors

Parent
Father
Mother
Sibling
Uncle

Male donors

Female-into-male allografting

Preparative regimen

Chemotherapy-only

Chemotherapy + TBI

GVHD prophylaxis

PTCy + tacrolimus + MMF

PTCy

Donor age, years

Median 30

Range $\quad 10-49$

CMV at risk

Total nucleated cells/kg IBW infused

$\begin{array}{ll}\text { Median } & 6.60 \times 10^{8} \\ \text { Range } & 2.06-22.8 \times 10^{8} \\ \text { Target cell dose } / \mathrm{kg} \mathrm{IBW} & 4 \times 10^{8} \\ \mathrm{CD} 34^{+} \text {cells } / \mathrm{kg} & \\ \text { Median } & 5.98 \times 10^{6}\end{array}$

Table 1 (continued)

\begin{tabular}{lll}
\hline Characteristic & Number & Percent \\
\hline Range & $2.45-16.2 \times 10^{6}$ & \\
ABO mismatch & & \\
Compatible & 17 & 68 \\
Major mismatch & 4 & 16 \\
Minor mismatch & 4 & 16 \\
\hline
\end{tabular}

$A B O$, blood group type; $A D A$, adenosine deaminase deficiency; Birc4, baculoviral IAP repeat-containing protein $4 ; B M T$, bone marrow transplantation; $C G D$, chronic granulomatous disease; $C M V$, cytomegalovirus; CTLA4, cytotoxic T lymphocyte-associated protein 4; CVID, common variable immune deficiency; $D B A$, Diamond-Blackfan anemia; $D K C$, dyskeratosis congenita; $G T$, Glanzmann's thrombasthenia; $G V H D$, graft-versus-host disease; $H L A$, human leukocyte antigen; $H L H$, hemophagocytic lymphohistiocytosis; $I B W$, ideal body weight; $I P E X$, immune dysregulation polyendocrinopathy X-linked; $M M F$, mycophenolate mofetil; PTCy, post-transplant cyclophosphamide; SCID, severe combined immune deficiency; STAT3, signal transducer and activator of transcription 3, XIAP, X-linked inhibitor of apoptosis

\section{Outcomes}

\section{Engraftment}

All patients achieved prompt neutrophil and platelet recovery. Neutrophil recovery occurred at a median of 16 days (range 13-33). Platelet recovery occurred at a median of 21 days (range 15-55). The median absolute lymphocyte counts (ALC) are depicted in Fig. 2. The median ALC at days 30, 60,180 , and 1 year were $0.38 \times 10^{9} / \mathrm{L}$ (range $0.02-1.14 \times 10^{9} /$ L), 0.60 (range $\left.0.18-2.22 \times 10^{9} / \mathrm{L}\right), 0.95($ range $0.14-2.10) \times$ $10^{9} / \mathrm{L}$, and $1.76 \times 10^{9} / \mathrm{L}$ (range $0.37-4.35 \times 10^{9} / \mathrm{L}$ ), respectively. Chimerism results are summarized in Tables 2 and 3 and depicted in Fig. 3. Notably, all 25 patients (100\%) had detectable donor chimerism at day 30; and 23 of 24 (96\%) evaluable patients had $\geq 95 \%$ donor chimerism in PB at day 30. In the patients with TBI-containing preps $(n=8), 6$ of 7 evaluable patients ( $86 \%$ ) had $\geq 95 \%$ donor chimerism in PB at 1 year, whereas in the patients with chemotherapy-only preps $(n=17), 7$ of 14 evaluable patients $(50 \%)$ had $\geq 95 \%$ donor chimerism in $\mathrm{PB}$ at 1 year $(p=0.11)$.

Of the 6 PIDD/PIRD patients treated with chemotherapy + TBI preps, there was one each of immune dysregulation polyendocrinopathy X-linked (IPEX), X-linked inhibitor of apoptosis (XIAP) deficiency, CGD, hyper-IgM syndrome, hemophagocytic lymphohistiocytosis (HLH), and common variable immune deficiency (CVID). Of these, four remain $100 \%$ donor, one had late secondary graft failure requiring a second BMT (Patient \#1), and one died of transplant-related mortality (Patient \#15). The two IBMFS patients treated with chemotherapy + TBI prep were both DKC patients, and both remain $100 \%$ donor at 4 and 7 years out from their BMTs. 


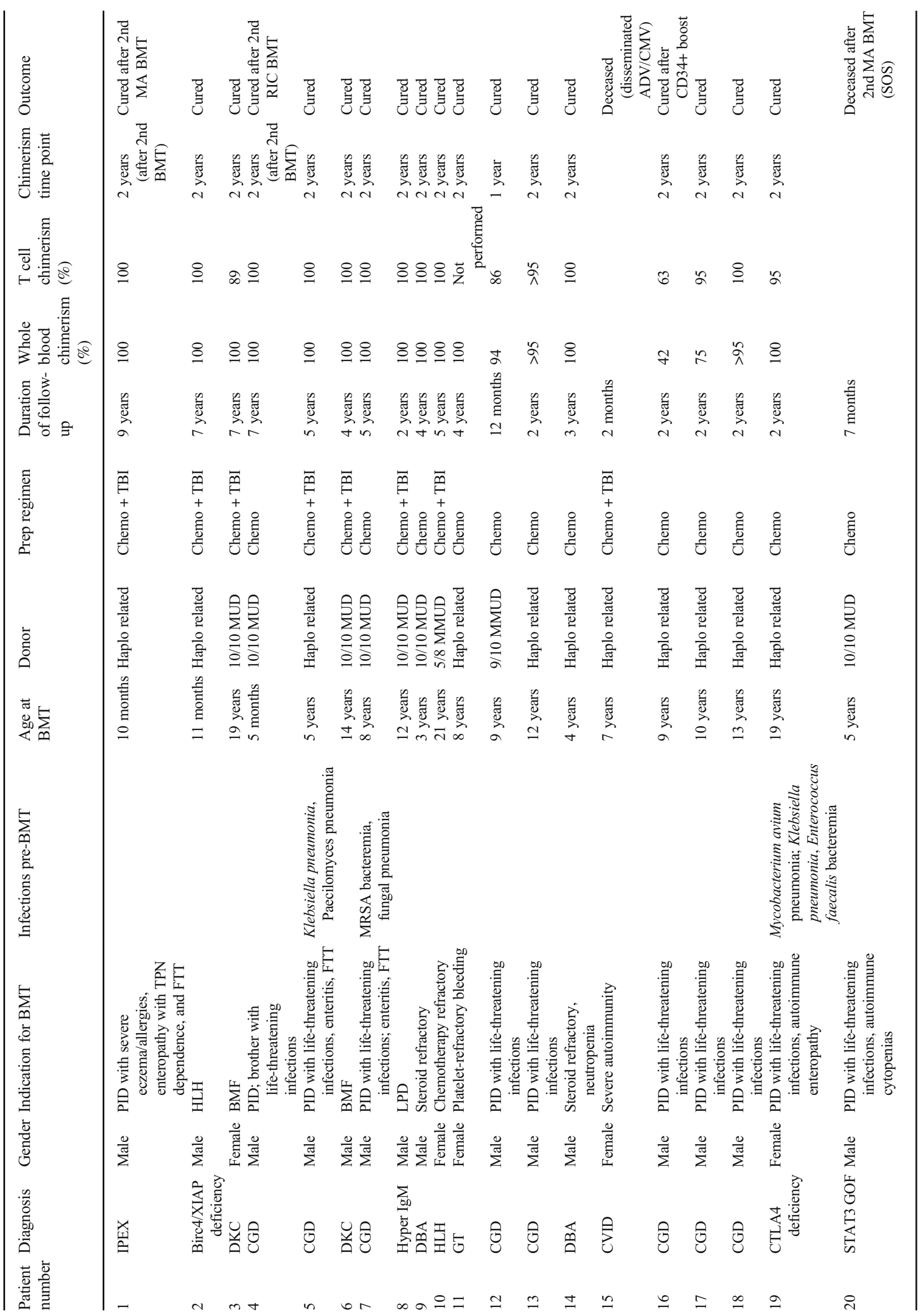




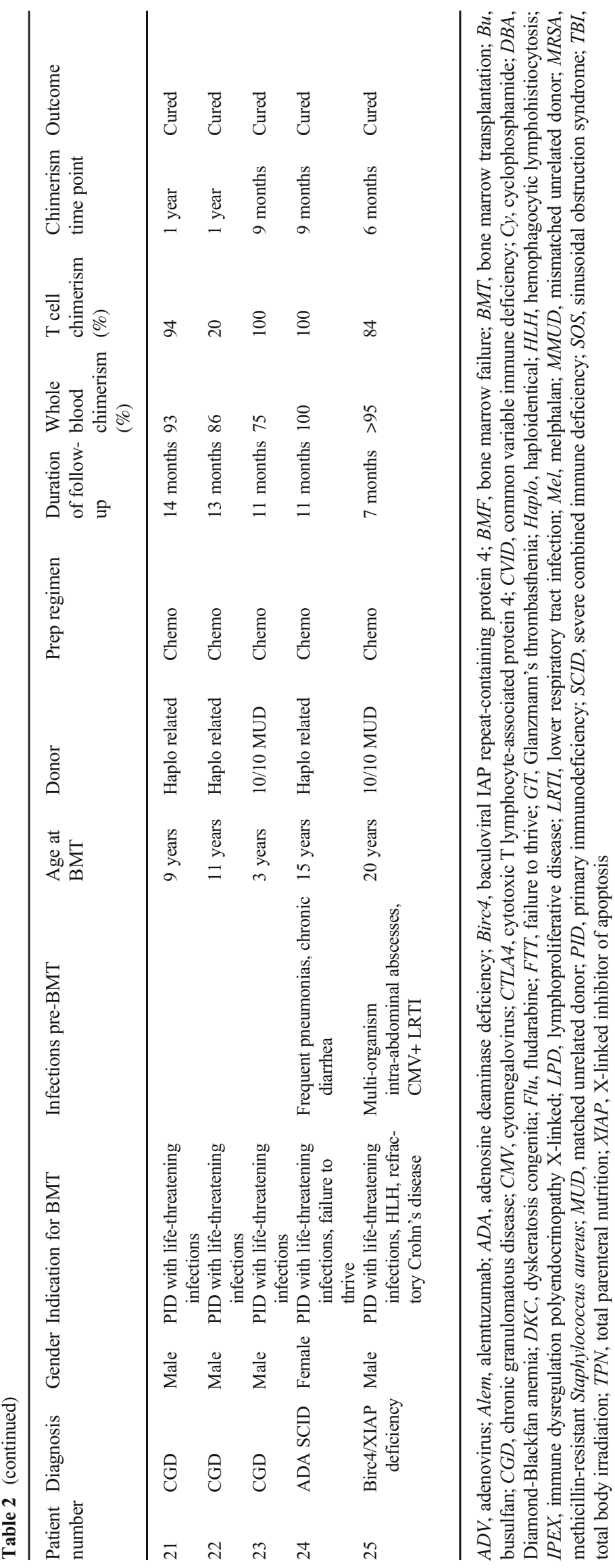




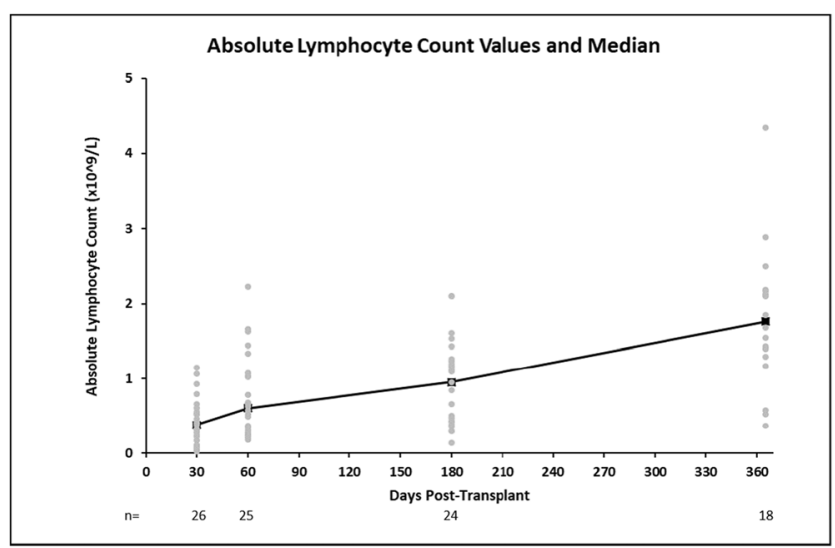

Fig. 2 Absolute lymphocyte count (ALC) values and median. The individual values for absolute lymphocyte count (ALC) are shown for days $30,60,180$, and 365. The median ALC at each time point were $0.38 \times$ $10^{9} / \mathrm{L}$ (range $0.02-1.14 \times 10^{9} / \mathrm{L}$ ), $0.60\left(\right.$ range $0.18-2.22 \times 10^{9} / \mathrm{L}$ ), 0.95 (range $0.14-2.10) \times 10^{9} / \mathrm{L}$, and $1.76 \times 10^{9} / \mathrm{L}\left(\right.$ range $0.37-4.35 \times 10^{9} / \mathrm{L}$ ), respectively. The total number of patients at each time point is $n=26, n=$ $25, n=24$, and $n=18$, respectively

Of the 14 PIDD/PIRD patients with chemo-only preps, 10 were patients with chronic granulomatous disease (CGD), and one each had signal transducer and activator of transcription 3 (STAT3) gain-of-function, adenosine deaminase deficiency (ADA) deficient severe combined immune deficiency (SCID), XIAP deficiency, and cytotoxic T lymphocyteassociated protein 4 (CTLA4) deficiency. Of the 10 CGD patients treated with chemotherapy-only, seven are mixed chimeras, one is $100 \%$ donor, one required CD34 boost (Patient \#16), and one had late graft failure and was successfully retransplanted (Patient \#4). Of the non-CGD PIDD/PIRD patients treated with chemotherapy-only, two are $100 \%$ donor, one is a mixed chimera, and one had early mixed chimerism and died after a second myeloablative BMT attempt (Patient \#20). Of the three IBMFS patients treated with chemo-only preps, two with Diamond-Blackfan anemia (DBA) and one with Glanzmann's thrombasthenia (GT), all are 100\% donor at 2 years post-BMT.

In the CGD cohort $(n=11)$, all patients had nitro blue tetrazolium testing (NBT), testing at day 30 or 60 to confirm resolution of the underlying disease. All eleven patients' testing revealed normal oxidative bursts. One patient (patient \#16), described below, did eventually develop CGD-related infections, requiring donor CD34 boost. Another CGD patient (patient \#4), had stable mixed chimerism for over 3 years, without any evidence of infection. At 3.5 years after BMT, with donor chimerism around $8 \%$, he did develop infections consistent with CGD, underwent a second RIC BMT from a different donor, and has been $100 \%$ donor \#2, now more than 2 years out from his second BMT. The remaining nine CGD patients have not had any CGD-related infections.

Four patients $(12 \%)$ ultimately required infusion of additional stem cell products. Two patients from our first cohort

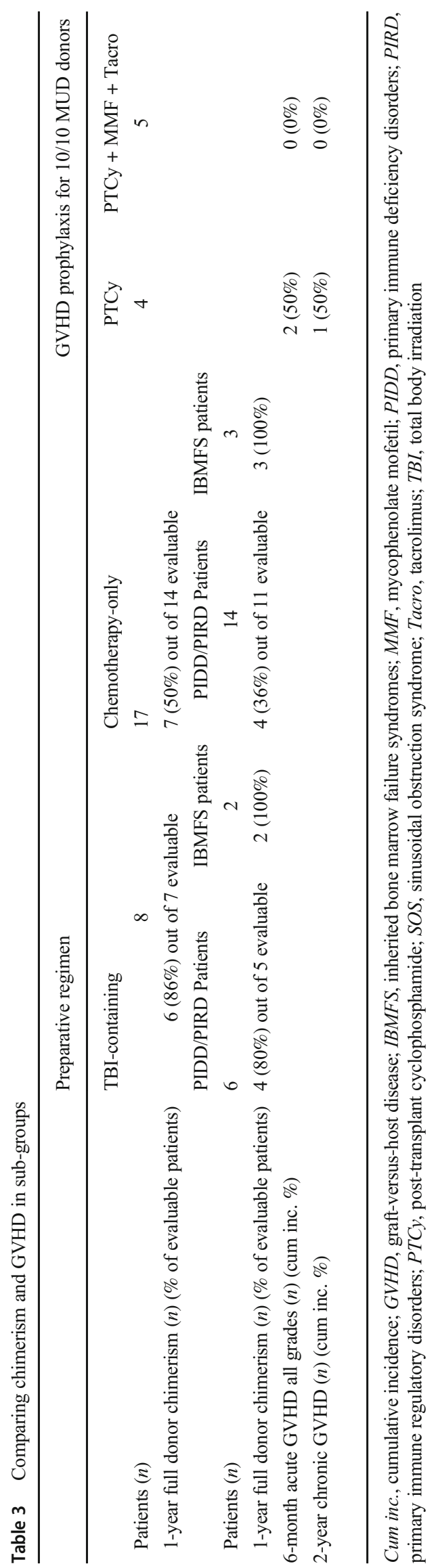




\section{- Full \\ $\square$ High-Level Mixed \\ 口Low-Level Mixed \\ $\square$ None detected \\ ○Not measured}
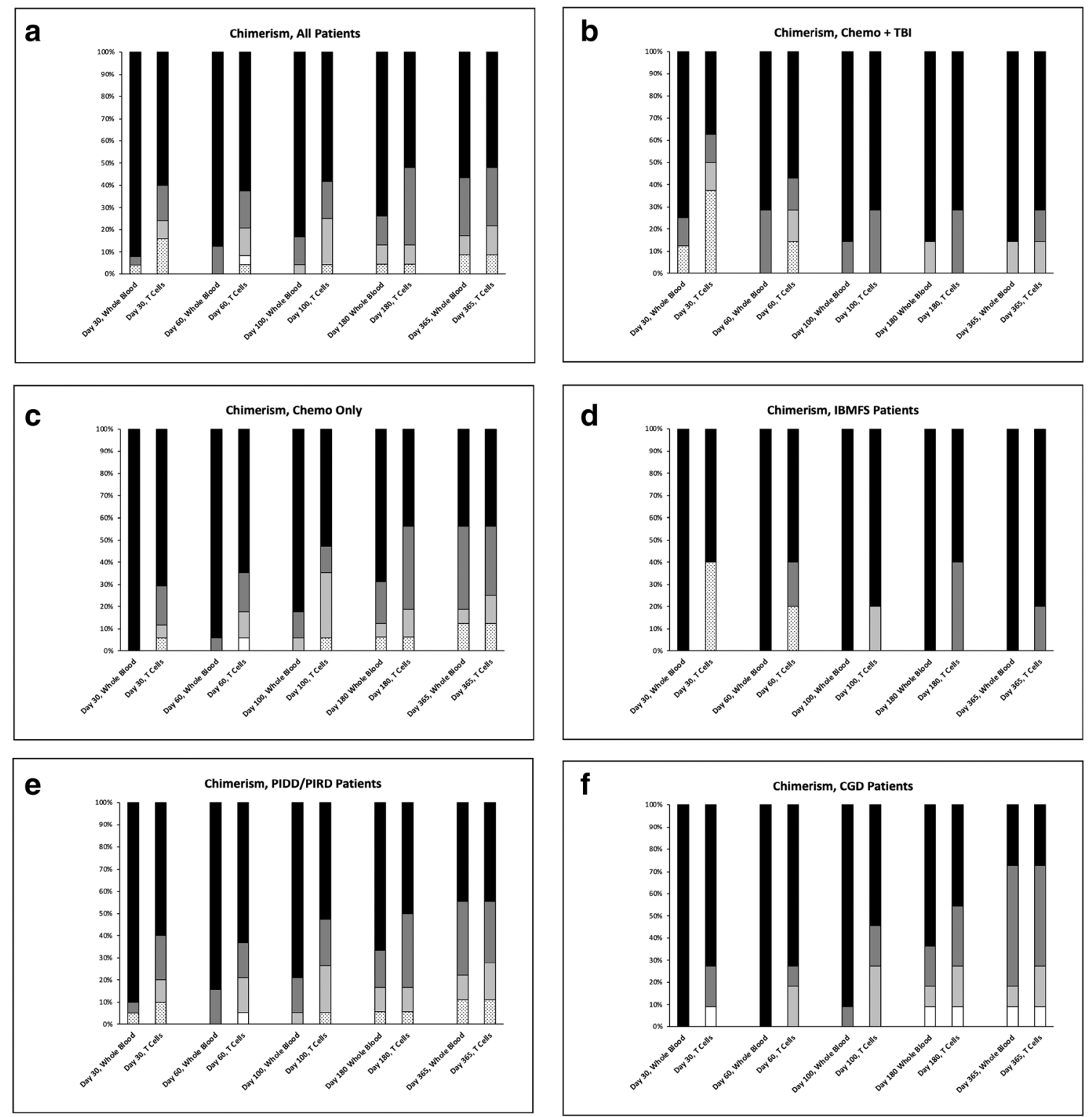

Fig. 3 Chimerism in whole blood and CD3+ compartment. Chimerism for all patients, $n=25$ (A), chemotherapy + total body irradiation, $n=8$ (B), Chemotherapy-only, $n=18$ (C), IBMFS patients, $n=5$ (D), PIDD/PIRD, $n=20$ patients (E), and CGD patients, $n=11$ (F). Graphs

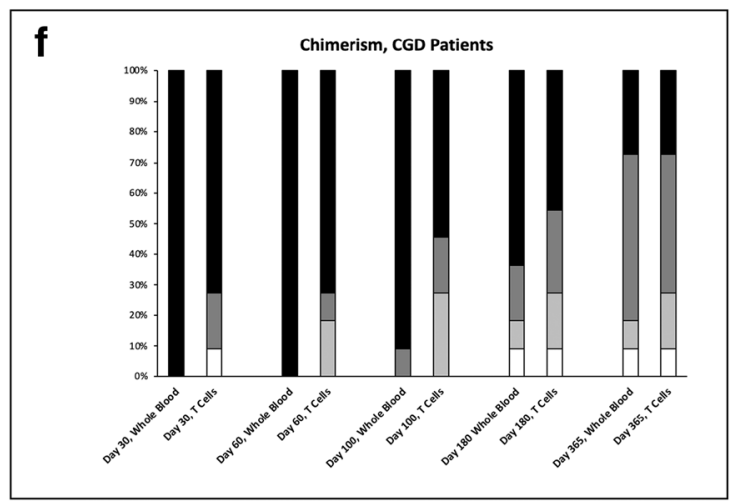

show percent of patients with full chimerism (defined as $>95 \%$ ), highlevel mixed (defined as 50-95\%), low-level mixed (defined as $<50 \%$ but detectable), none detected, or not measured

[8] experienced late secondary graft failure, patient \#1 with IPEX (TBI-containing prep) and patient \#4 with GGD (chemotherapy-only prep), one patient with CGD (\#16) (chemotherapy-only prep) was treated with a $\mathrm{CD} 34^{+}$boost

at 2 years post-BMT, and one patient with STAT3 GOF (\#20) (chemotherapy-only prep) had early secondary graft failure, was treated with a myeloablative BMT, and ultimately died from transplant-related complications. 
Patient \#1 had detectable host as early as day 30 after BMT, with BM chimerism of $94 \%$, and had a slow decline of donor chimerism over time. He had whole blood and CD3 chimerism of 5-8\% donor at 1,2, and 3 years after BMT, but no symptoms of his underlying disease. He developed an acute gastroenteritis and subsequently developed symptoms of IPEX. He was re-transplanted with the same haploidentical donor using a myeloablative regimen, and is alive, $100 \%$ donor, and free of IPEX-symptoms at 6 years since his second BMT. Patient \#4 was $100 \%$ donor in whole blood at day 30 , then had detectable host at day 60 with whole blood chimerism of $95 \%$. He had a slow decline in donor chimerism, and had mixed chimerism in the $10-15 \%$ range in both whole blood and T cells at 1,2, and 3 years after BMT, but no symptoms of CGD. At around 3.5 years after BMT, he developed retinitis, and repeat NBT testing showed no oxidative burst. He was re-transplanted using a busulfan-based RIC regimen using a haploidentical donor. He is alive, $100 \%$ donor, and has had no CGD-related infections at more than 2 years since his second BMT.

Patient \#16 with CGD developed detectable host T cells at day +60 and remained a mixed chimera with declining donor for 2 years. Repeat NBT testing revealed defective oxidative burst of $6 \%$, and he was treated with an unprepped CD $34^{+}$ selected stem cell boost from his haploidentical donor, with a cell dose of $31.2 \times 10^{6} \mathrm{TNC} / \mathrm{kg}$ and $30.5 \times 10^{6} \mathrm{CD} 34^{+} / \mathrm{kg}$. His subsequent percent donor chimerism rose, and repeat NBT testing revealed a normal oxidative burst of $63 \%$. He has remained mixed chimerism, and free of CGD-related infections at 10 months since the CD34 boost. Patient \#20, with STAT3 GOF mutation, had $>95 \%$ donor in $\mathrm{WB}$ and $20 \%$ in TCs at day 30 . By day 60 , the WB was $68 \%$ donor, with no detectable donor TCs. He developed symptoms of his underlying disease, and was re-transplanted 6 months later using a myeloablative regimen and the same donor. He achieved count recovery and donor chimerism of $100 \%$ in $\mathrm{WB}$ and > $95 \%$ in TCs by day 30 , but unfortunately died from severe sinusoidal obstruction syndrome.

\section{Graft-Versus-Host Disease}

The 6-month cumulative incidence of grade II-IV aGVHD is $17 \%$, with no cases of grades III or IV aGVHD. Six patients developed grades I $(n=2)$ or II $(n=4)$ aGVHD, and all responded to steroids and a calcineurin inhibitor. In patients who received MUD donors and PTCy alone $(n=4)$, two developed skin-only acute GVHD, one grade I and one grade II. In patients who received MUD donors and PTCy, MMF, and tacrolimus $(n=5)$, none developed acute GVHD. The 1-year cumulative incidence of any cGVHD is $14 \%$, with severe cGVHD of 5\%. Three patients developed cGVHD, two with mild limited, and one with severe extensive. In patients who received MUD donors and PTCy alone $(n=4)$, one developed mild limited chronic GVHD. In patients who received MUD donors and PTCy, MMF, and tacrolimus $(n=5)$, none developed chronic GVHD. Both patients with mild cGVHD were treated, and are off systemic immune suppression. One patient (\#24) developed early, gut-only, grade II aGVHD, which responded to steroids and sirolimus. She later developed extensive cGVHD, including autoimmune cytopenias (see below), at 11 months post-transplant. As of this writing, she has responded to treatment with steroids and sirolimus with improvement of symptoms, and steroids are being weaned. Cumulative incidences of GVHD for different GVHD prophylaxis regimens with MUD donors are shown in Table 3.

\section{Survival}

The median follow-up of surviving patients is 26 months (ranging 7 months -9 years) (Table 2). Overall survival and disease-free survival at 2 years are both $92 \%$ (95\% CI 72 98\%). Event-free survival at 2 years is $87 \%$ (95\% CI 64 96\%) (Fig. 4). For the 17 surviving patients who have follow-up of or beyond 2 years, the OS is $90 \%$ (95\% CI 64 $97 \%$ ) and the EFS is $68 \%$ (95\% CI 38-86\%). The 2-year OS and EFS in the TBI-containing versus chemotherapy-only preparative regimens are not statistically significantly different.

\section{Transplant-Related Complications}

Sinusoidal Obstruction Syndrome Three patients (12\%) developed SOS, as defined by Baltimore criteria [17]. Two had severe sinusoidal obstruction syndrome (SOS) with multiorgan dysfunction (MOD) and were successfully treated with defibrotide (DF). One of these patients received TBI in the prep, and one had a chemotherapy-only prep. As described above, patient \#20 developed fatal SOS with MOD following a second myeloablative BMT. Notably, patient \#25 had a history of poorly controlled Crohn's and multiple intraabdominal fistulae. He was treated with prophylactic DF from the start of his prep to day +30 , per a recently published phase

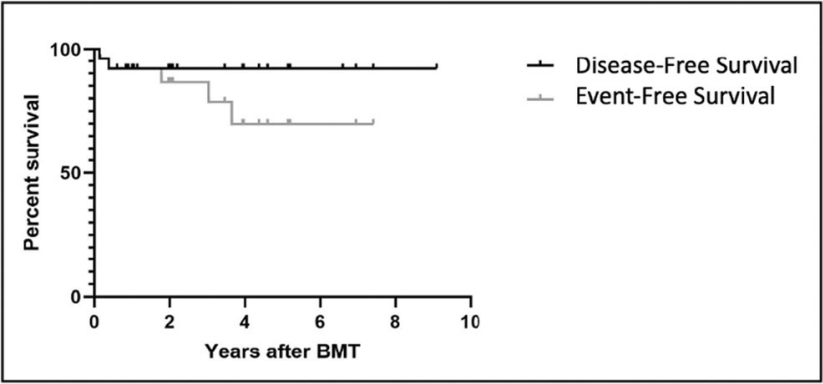

Fig. 4 Disease-free survival (DFS)/overall survival (OS) and event-free survival (EFS). Disease-free survival/overall survival and event-free survival for all patients. OS and DFS are the same at 2 years; three patients who developed secondary graft failure were successfully treated 
3 trial [18], and did not develop any signs or symptoms of SOS.

Infections There was one mortality from infection. Patient \#15 with common variable immune deficiency (CVID) developed concurrent adenovirus and CMV infections early after BMT following initial count recovery. This ultimately led to secondary graft failure, multi-organ failure, and death before day + 60 despite targeted anti-viral therapy and advanced supportive care.

There were two patients $(8 \%)$ with CMV reactivation, two patients $(8 \%)$ with BK hemorrhagic cystitis, two patients $(8 \%)$ with lower respiratory tract influenza, three patients $(12 \%)$ with EBV viremia, and one patient (4\%) with HHV-6 viremia. All viral infections/reactivations occurred within the first 6 months after BMT. Four patients (16\%) had Clostridium difficile-positive diarrhea, there were thirteen episodes of bacteremia in nine patients (36\%), and one patient (4\%) had a urinary tract infection. Patient \#19 with CTLA deficiency had multiple episodes of bacteremia in the first year after BMT; the remaining bacterial infections in other patients occurred within the first 3 months after BMT. One patient (4\%) had a fungal pneumonia, which occurred at day +48 after BMT.

Autoimmune Cytopenias/Inflammation Three patients (12\%) developed autoimmune cytopenias. All three had chemotherapy-only prep regimens, and all three received PTCy, MMF, and tacrolimus for GVHD prophylaxis. One patient (\#21) developed direct antibody test (DAT)-positive hemolytic anemia at 10 months post-BMT. He was treated successfully with a high-dose steroid pulse followed by a taper. One patient (\#22) developed unexplained neutropenia at 6 months post-BMT. He was treated with high-dose pooled intravenous immunoglobulin (IVIg), and his ANC rose to normal. Patient \#24 had persistent unexplained thrombocytopenia accompanied by DAT-positive hemolytic anemia at 10 months post-BMT. She was treated with IVIg with a slight rise in her platelet count and hemoglobin. She subsequently developed fevers and dysregulated immunity - consisting of elevated ferritin, erythrocyte sedimentation rate, and triglycerides - and ultimately both skin and gastrointestinal biopsy-proven cGVHD. Her cytopenias and inflammatory markers improved after initiating cGVHD-directed therapy.

Cytokine Release Syndrome Cytokine release syndrome has been reported at high rates in recipients of haploidentical PBSC grafts, and at much lower rates with haploidentical $\mathrm{T}$ cell replete bone marrow [19, 20]. Based on standard cytokine release syndrome (CRS) grading criteria [21], none of the patients developed CRS. The patient who received a PBSC graft developed a fever after the infusion, but no other signs or symptoms of CRS.

\section{Discussion}

We provide an updated report of pediatric patients with PIDD, PIRD, and IBMFS treated with RIC alloBMT and PTCy for GVHD prophylaxis. We added 14 new patients who did not receive TBI and who received post-transplant $\mathrm{MMF}$ and tacrolimus, including 6 with rare diseases, and we report longerterm follow-up for the entire cohort. This study adds significant clinical equipoise, particularly for alloBMT using donors other than HLA-matched related donors. Our results using a uniform RIC preparative regimen demonstrate 2-year overall and event-free survivals of $92 \%$ and $87 \%$, respectively, and for the patients who have follow-up of or beyond 2 years, we demonstrate overall and event-free survivals of $90 \%$ and $68 \%$, respectively. We demonstrate cumulative incidences of aGVHD grade II to IV of $17 \%$, with no grade III or IV aGVHD, cGVHD of 14\%, severe cGVHD of 5\%, and 1year TRM of $4 \%$. We also demonstrate robust median ALC recovery $[22,23]$, rapid neutrophil engraftment, and low rates of infectious complications, in the setting of limited rates of acute and chronic GVHD.

Based on our initial successful rates of early robust donor chimerism in our first eleven patients [8], we removed lowdose TBI from the preparative regimen for all but one of the additionally reported 14 patients. Since making this change, we have noted an increase in mixed chimerism in PIDD/PIRD patients, more predominantly in the CGD patients. By contrast, removal of TBI from the prep has not affected chimerism in the IBMFS patients, though it is unclear whether this is significant given the smaller number of IBMFS patients treated with chemotherapy-only $(n=4)$. The surviving PIDD/ PIRD mixed chimera patients have no symptoms of their underlying diseases, with follow-up ranging 9 months-3.5 years, off immune suppression, and at varying percentages of donor chimerism. In many PIDD/PIRD patients, stable mixed chimerism may be sufficient for long-term cure, but these patients may be at risk for late graft failure. This is especially relevant for diseases where the defect does not impair $\mathrm{T}$ cell function, such as in CGD, wherein mixed chimerism in neutrophils, even with a low donor percentage, may be enough to cure the underlying disease. Based on the PIDD and PIRD patient outcomes, we believe that low-dose TBI in the conditioning regimen is more likely to result in full donor chimerism, thereby preventing late graft failure that may occur with mixed chimerism. We have therefore added TBI back into the regimen for all PIDD/PIRD patients only, but not for IBMFS patients, and are studying this in a prospective institutional trial (ClinicalTrials.gov Identifier: NCT04232085).

PTCy has been used successfully as sole GVHD prophylaxis in the matched donor setting for hematologic malignancies [24-26]. In our first publication [8], we used PTCy alone as GVHD prophylaxis in four patients with 10/10 MUDs. Two developed aGVHD, and one developed cGVHD. In an 
attempt to further minimize GVHD and optimize engraftment in our additional patients, we added MMF and tacrolimus to PTCy for GVHD prophylaxis for all patients, regardless of degree of donor match, as was recently shown to be effective in a large study of patients with hematologic malignancies [27]. None of the five patients transplanted using MUDs and PTCy, MMF, and tacrolimus developed GVHD. Using PTCy, MMF, and tacrolimus for all donor types was successful in limiting acute and chronic GVHD.

Mallhi et al. recently published the outcomes of a similarly heterogeneous group of 23 patients with nonmalignant disorders, transplanted with a cyclophosphamide-based RIC regimen, and similar GVHD prophylaxis [9]. Four (17\%) of their patients only received one dose of PTCy, and $26 \%$ received PBSC grafts. Their 2-year OS and EFS are excellent, $91 \%$ and $78 \%$, but their rates of acute and chronic GVHD are much higher than other reported PTCy regimens [8, 28-31]. They observed acute GVHD grades II-IV and III-IV and chronic GVHD rates of $78 \%, 26 \%$, and $42 \%$ [9], whereas in our cohort, those rates were $17 \%, 0 \%$, and $14 \%$. We have demonstrated similar OS and EFS with significantly lower GVHD rates.

Allen et al. recently published the outcomes from the BMT CTN 1204 study, wherein 46 patients with HLH or PIDD were transplanted using RIC and cyclosporine-based GVHD prophylaxis [7]. They reported 18-month OS 67\%, grade IIIV aGVHD $26 \%$, and cGVHD of $27 \%$ [7]. Unfortunately, mortality and graft failure remained problematic; two patients (4\%) had primary graft failure, and the probability of being alive at 1 year, with donor chimerism significant to cure, and without any additional intervention, was 39\% [7]. We recognize that this study had a much higher percentage of HLH patients than ours, which impedes any direct comparison.

In a more comparable patient population, Dimitrova et al. recently published the outcomes of twenty PIDD patients transplanted with serotherapy-free chemotherapy-only RIC and PTCy [32]. The outcomes for this study were 1-year OS 90\%, grade II-IV aGVHD 20\%, and no cGVHD; however, only $45 \%$ were alive and without additional infusion of stem cells at 1 year.

An additional notable comparison is provided by Gungor et al., who reported the outcomes of 56 CGD patients transplanted using busulfan-based RIC and CNI/MMF for GVHD prophylaxis [33]. The cumulative incidence of aGVHD grade II-IV was $11 \%$ and III-IV was $4 \%$, and cGVHD of $0 \%$ with matched siblings and $11 \%$ with unrelated donors [33]. In this study, the probability of being alive and without infusion of additional stem cells at 1 year was $91 \%$; later probabilities are not reported. In our CGD cohort $(n=$ 11), two of our CGD patients did require infusion of additional stem cell products at later time points, resulting in the probability of being alive and without infusion of additional stem cells at 4 years of $82 \%$. It is important to note that haploidentical donors were not studied in the Gungor report [33]; our successful use of PTCy in the RIC setting further expands the donor pool for those who do not have unrelated donors, while maintaining comparably high rates of engraftment at 1 year and low rates of GVHD.

An alternative approach to PTCy is ex vivo T cell receptor $\alpha \beta+$ and CD19+ cell depletion, which has been used in both the malignant and nonmalignant settings [34-37]. Shah et al. transplanted 25 children with PIDD using RIC and MMF/ cyclosporine for GVHD prophylaxis [36]. OS and EFS at 3 years were $84 \%$ and $80 \%$. Cumulative incidence of grade II-IV aGVHD was $22 \%$, with no cGVHD, and TRM at 1 year was $16 \%$. Ex vivo T cell depletion has many similar benefits to PTCy, in that it widely expands the donor pool and allows for decreased use of immune suppression after BMT. However, the cost of $\mathrm{T}$ cell depletion is high compared to unmanipulated strategies [38], and it is only available at centers with graft engineering expertise, thus making it less accessible.

We acknowledge the heterogeneity in our patient population, with an over-representation of CGD patients, an underrepresentation of HLH patients, and small numbers of IBMFS patients. Patients with PIDD/PIRD have a variety of underlying defects leading to chronic inflammation and chronic infection, leading to organ damage and reduced performance status, and ultimately to decreased life expectancy. Though varying in their defects, we believe there is benefit in treating all of these patients with a one-size-fits-all regimen, in order to simplify the regimen to make it accessible to patients with all diagnoses and with any donor type. Though eleven of our 25 patients $(44 \%)$ have CGD, we believe the results herein are applicable to other PIDD/PIRD diagnoses. However, it is important to examine this in a prospective manner, as we are doing in our recently opened phase 2 ongoing institutional trial (ClinicalTrials.gov Identifier: NCT04232085), in order to more closely study engraftment and the kinetics of immune reconstitution for all PIDD, PIRD, and IBMFS patients.

\section{Conclusions}

Our results utilizing RIC with PTCy compare favorably to all of these studies, with low rates of GVHD and high rates of survival. All patients had prompt count recovery and no patients experienced primary graft failure. Though we have observed an increase in mixed chimerism since removing lowdose TBI from the preparative regimen, disease-free survival at 1 year, without infusion of additional stem cell products is $91 \%$ ( 21 of 23 evaluable patients). Three of the 21 patients did ultimately need additional stem cell products at time points later than 1 year. While this is a smaller, single-institution retrospective study with a skew towards CGD patients and 
fewer HLH patients than some of the comparable data $[4,7$, 39], our results are an important addition to the field, as more centers develop regimens with the goals of minimizing toxicity and improving engraftment. RIC with PTCy provides a platform for the safe and effective use of HLA-matched or mismatched unrelated donors and haploidentical related donors, and should be considered a curative therapeutic option for patients with IBMFS, PIDD, and PIRD.

Acknowledgements The authors would like to thank the patients and their families, as well as the physicians, advanced practice providers, nurses, care managers, transplantation coordinators, and other providers and staff members in the Pediatric Blood and Marrow Transplantation Program at The Johns Hopkins Hospital Sidney Kimmel Comprehensive Cancer Center who participated in the care of these patients. The authors would also like to thank the Cell Therapy Laboratory staff for providing graft composition data on the patients studied.

\section{Compliance with Ethical Standards}

Conflict of Interest K.R.C. serves on the Speaker Bureau and Advisory Board for Jazz pharmaceuticals and received a research grant from Jazz pharmaceuticals; he is on an investigator-initiated trial of post-transplant therapy for solid tumors that is supported in part by Bristol Meyers Squibb. H.J.S. is on the Speaker Bureau for Jazz pharmaceuticals and is on an investigator-initiated trial of post-transplant therapy for solid tumors that is supported in part by Bristol Meyers Squibb. None of these are in direct conflict with the manuscript.

\section{References}

1. Gragert L, Eapen M, Williams E, Freeman J, Spellman S, Baitty R, et al. HLA match likelihoods for hematopoietic stem-cell grafts in the U.S. Registry. N Engl J Med. 2014;371(4):339-48.

2. Filipovich AH, Stone JV, Tomany SC, Ireland M, Kollman C, Pelz $\mathrm{CJ}$, et al. Impact of donor type on outcome of bone marrow transplantation for Wiskott-Aldrich syndrome: collaborative study of the International Bone Marrow Transplant Registry and the National Marrow Donor Program. Blood. 2001;97(6):1598-603.

3. Antoine C, Muller S, Cant A, Cavazzana-Calvo M, Veys P, Vossen $\mathrm{J}$, et al. Long-term survival and transplantation of haemopoietic stem cells for immunodeficiencies: report of the European experience 1968-99. Lancet. 2003;361(9357):553-60.

4. Marsh RA, Rao MB, Gefen A, Bellman D, Mehta PA, Khandelwal $\mathrm{P}$, et al. Experience with alemtuzumab, fludarabine, and melphalan reduced-intensity conditioning hematopoietic cell transplantation in patients with nonmalignant diseases reveals good outcomes and that the risk of mixed chimerism depends on underlying disease, stem cell source, and alemtuzumab regimen. Biol Blood Marrow Transplant. 2015;21(8):1460-70.

5. Hagin D, Burroughs L, Torgerson TR. Hematopoietic stem cell transplant for immune deficiency and immune dysregulation disorders. Immunol Allergy Clin N Am. 2015;35(4):695-711.

6. Dinardo L, Brown V, Perez E, Bunin N, Sullivan KE. A singlecenter study of hematopoietic stem cell transplantation for primary immune deficiencies (PIDD). Pediatr Transplant. 2012;16(1):6372.

7. Allen CE, Marsh R, Dawson P, Bollard CM, Shenoy S, Roehrs P, et al. Reduced-intensity conditioning for hematopoietic cell transplant for HLH and primary immune deficiencies. Blood. 2018;132(13):1438-51.
8. Klein OR, Chen AR, Gamper C, Loeb D, Zambidis E, Llosa N, et al. Alternative-donor hematopoietic stem cell transplantation with post-transplantation cyclophosphamide for nonmalignant disorders. Biol Blood Marrow Transplant. 2016;22(5):895-901.

9. Mallhi KK, Srikanthan MA, Baker KK, Frangoul HA, Torgerson TR, Petrovic A, et al. HLA-haploidentical hematopoietic cell transplantation for treatment of nonmalignant diseases using nonmyeloablative conditioning and post-transplant cyclophosphamide. Biol Blood Marrow Transplant. 2020;26(7):1332-41.

10. Rao K, Adams S, Qasim W, Allwood Z, Worth A, Silva J, et al. Effect of stem cell source on long-term chimerism and event-free survival in children with primary immunodeficiency disorders after fludarabine and melphalan conditioning regimen. J Allergy Clin Immunol. 2016;138(4):1152-60.

11. Przepiorka D, Weisdorf D, Martin P, Klingemann H, Beatty P, Hows J, et al. 1994 Consensus conference on acute GVHD grading. Bone Marrow Transplant. 1995;6(15):825-8.

12. Jagasia MH, Greinix HT, Arora M, Williams KM, Wolff D, Cowen EW, et al. National Institutes of Health Consensus development project on criteria for clinical trials in chronic graft-versus-host disease: I. The 2014 Diagnosis and Staging Working Group report. Biol Blood Marrow Transplant. 2015;21(3):389-401.e1.

13. Filipovich AH, Weisdorf D, Pavletic S, Socie G, Wingard JR, Lee SJ, et al. National Institutes of Health Consensus development project on criteria for clinical trials in chronic graft-versus-host disease: I. Diagnosis and Staging Working Group report. Biol Blood Marrow Transplant. 2005;11(12):945-56.

14. De Pauw B, Walsh TJ, Donnelly JP, Stevens DA, Edwards JE, Calandra $T$, et al. Revised definitions of invasive fungal disease from the European Organization for Research and Treatment of Cancer/Invasive Fungal Infections Cooperative Group and the National Institute of Allergy and Infectious Diseases Mycoses Study Group (EORTC/MSG) Consensus Group. Clin Infect Dis. 2008;46(12):1813-21.

15. Kaplan E, Meier P. Nonparametric estimation from incomplete observations. J Am Stat Assoc. 1958;53:457-80.

16. Gooley TA, Leisenring W, Crowley J, Storer BE. Estimation of failure probabilities in the presence of competing risks: new representations of old estimators. Stat Med. 1999;18(6):695-706.

17. Jones R, Lee K, Beschorner W, Vogel V, Grochow L, Braine H, et al. Venoocclusive disease of the liver following bone marrow transplantation. Transplantation. 1987;6(44):778-83.

18. Corbacioglu S, Cesaro S, Faraci M, Valteau-Couanet D, Gruhn B, Rovelli A, et al. Defibrotide for prophylaxis of hepatic venoocclusive disease in paediatric haemopoietic stem-cell transplantation: an open-label, phase 3, randomised controlled trial. Lancet. 2012;379(9823):1301-9.

19. Imus PH, Blackford AL, Bettinotti M, Luznik L, Fuchs EJ, Huff CA, et al. Severe cytokine release syndrome after haploidentical peripheral blood stem cell transplantation. Biol Blood Marrow Transplant. 2019;25(12):2431-37

20. McCurdy SR, Muth ST, Tsai HL, Symons HJ, Huff CA, Matsui WH, et al. Early fever after haploidentical bone marrow transplantation correlates with class II HLA-mismatching and myeloablation but not outcomes. Biol Blood Marrow Transplant. 2018;24(10): 2056-64.

21. Lee DW, Gardner R, Porter DL, Louis CU, Ahmed N, Jensen M, et al. Current concepts in the diagnosis and management of cytokine release syndrome. Blood. 2014;124(2):188-95.

22. Kim HT, Armand P, Frederick D, Andler E, Cutler C, Koreth J, et al. Absolute lymphocyte count recovery after allogeneic hematopoietic stem cell transplantation predicts clinical outcome. Biol Blood Marrow Transplant. 2015;21(5):873-80.

23. Bayraktar UD, Milton DR, Guindani M, Rondon G, Chen J, AlAtrash G, et al. Optimal threshold and time of absolute lymphocyte 
count assessment for outcome prediction after bone marrow transplantation. Biol Blood Marrow Transplant. 2016;22(3):505-13.

24. Jacoby E, Chen A, Loeb DM, Gamper CJ, Zambidis E, Llosa NJ, et al. Single-agent post-transplantation cyclophosphamide as graftversus-host disease prophylaxis after human leukocyte antigenmatched related bone marrow transplantation for pediatric and young adult patients with hematologic malignancies. Biol Blood Marrow Transplant. 2016;22(1):112-8.

25. McCurdy SR, Kanakry CG, Tsai HL, Gojo I, Smith BD, Gladstone $\mathrm{DE}$, et al. Development of grade II acute graft-versus-host disease is associated with improved survival after myeloablative HLAmatched bone marrow transplantation using single-agent posttransplant cyclophosphamide. Biol Blood Marrow Transplant. 2019;25(6):1128-35.

26. Luznik L, Bolaños-Meade J, Zahurak M, Chen AR, Smith BD, Brodsky R, et al. High-dose cyclophosphamide as single-agent, short-course prophylaxis of graft-versus-host disease. Blood. 2010;115(16):3224-30.

27. Bolaños-Meade J, Reshef R, Fraser R, Fei M, Abhyankar S, AlKadhimi Z, et al. Three prophylaxis regimens (tacrolimus, mycophenolate mofetil, and cyclophosphamide; tacrolimus, methotrexate, and bortezomib; or tacrolimus, methotrexate, and maraviroc) versus tacrolimus and methotrexate for prevention of graft-versushost disease with haemopoietic cell transplantation with reducedintensity conditioning: a randomised phase 2 trial with a nonrandomised contemporaneous control group (BMT CTN 1203). Lancet Haematol. 2019;6(3):e132-e43.

28. Bolanos-Meade J, Fuchs EJ, Luznik L, Lanzkron SM, Gamper CJ, Jones RJ, et al. HLA-haploidentical bone marrow transplantation with posttransplant cyclophosphamide expands the donor pool for patients with sickle cell disease. Blood. 2012;120(22):4285-91

29. Bonfim C, Ribeiro L, Nichele S, Loth G, Bitencourt M, Koliski A, et al. Haploidentical bone marrow transplantation with posttransplant cyclophosphamide for children and adolescents with Fanconi anemia. Biol Blood Marrow Transplant. 2017;23(2):310 7.

30. DeZern AE, Zahurak M, Symons H, Cooke K, Jones RJ, Brodsky RA. Alternative donor transplantation with high-dose post-transplantation cyclophosphamide for refractory severe aplastic anemia. Biol Blood Marrow Transplant. 2017;23(3):498-504.

31. Luznik L, O'Donnell PV, Symons HJ, Chen AR, Leffell MS, Zahurak M, et al. HLA-haploidentical bone marrow transplantation for hematologic malignancies using nonmyeloablative conditioning and high-dose, posttransplantation cyclophosphamide. Biol Blood Marrow Transplant. 2008;14(6):641-50.
32. Dimitrova D, Gea-Banacloche J, Steinberg SM, Sadler JL, Hicks SN, Carroll E, et al. Prospective study of a novel, radiation-free, reduced-intensity bone marrow transplantation platform for primary immunodeficiency diseases. Biol Blood Marrow Transplant. 2020;26(1):94-106.

33. Gungor T, Teira P, Slatter M, Stussi G, Stepensky P, Moshous D, et al. Reduced-intensity conditioning and HLA-matched haemopoietic stem-cell transplantation in patients with chronic granulomatous disease: a prospective multicentre study. Lancet. 2014;383(9915):436-48.

34. Bertaina A, Zecca M, Buldini B, Sacchi N, Algeri M, Saglio F, et al. Unrelated donor vs HLA-haploidentical $\alpha / \beta$ T-cell- and B-celldepleted HSCT in children with acute leukemia. Blood. 2018;132(24):2594-607.

35. Rubio MT, D'Aveni-Piney M, Labopin M, Hamladji R-M, Sanz MA, Blaise D, et al. Impact of in vivo T cell depletion in HLAidentical allogeneic stem cell transplantation for acute myeloid leukemia in first complete remission conditioned with a fludarabine ivbusulfan myeloablative regimen: a report from the EBMT Acute Leukemia Working Party. J Hematol Oncol. 2017;10(1):31.

36. Shah RM, Elfeky R, Nademi Z, Qasim W, Amrolia P, Chiesa R, et al. T-cell receptor $\alpha \beta(+)$ and $C D 19(+)$ cell-depleted haploidentical and mismatched hematopoietic stem cell transplantation in primary immune deficiency. J Allergy Clin Immunol. 2018;141(4):1417-26.e1.

37. Balashov D, Shcherbina A, Maschan M, Trakhtman P, Skvortsova Y, Shelikhova L, et al. Single-center experience of unrelated and haploidentical stem cell transplantation with $\mathrm{TCR} \alpha \beta$ and CD19 depletion in children with primary immunodeficiency syndromes. Biol Blood Marrow Transplant. 2015;21(11):1955-62.

38. Patriarca F, Luznik L, Medeot M, Zecca M, Bacigalupo A, Di Bartolomeo P, et al. Experts' considerations on HLAhaploidentical stem cell transplantation. Eur J Haematol. 2014;93(3):187-97.

39. Parikh SH, Mendizabal A, Benjamin CL, Komanduri KV, Antony $\mathrm{J}$, Petrovic A, et al. A novel reduced-intensity conditioning regimen for unrelated umbilical cord blood transplantation in children with nonmalignant diseases. Biol Blood Marrow Transplant. 2014;20(3):326-36

Publisher's Note Springer Nature remains neutral with regard to jurisdictional claims in published maps and institutional affiliations. 